\title{
Urgences
}

\section{Entre les cases (quelques effets fictionnels des interstices en bande dessinée)}

\section{Richard Saint-Gelais}

Numéro 32, mai 1991

Lectures de bandes dessinées

URI : https://id.erudit.org/iderudit/025644ar

DOI : https://doi.org/10.7202/025644ar

Aller au sommaire du numéro

Éditeur(s)

Urgences

ISSN

0226-9554 (imprimé)

1927-3924 (numérique)

Découvrir la revue

Citer cet article

Saint-Gelais, R. (1991). Entre les cases (quelques effets fictionnels des interstices en bande dessinée). Urgences, (32), 7-17. https://doi.org/10.7202/025644ar d'utilisation que vous pouvez consulter en ligne.

https://apropos.erudit.org/fr/usagers/politique-dutilisation/ 


\section{Entre les cases (quelques effets fictionnels des interstices en bande dessinée) \\ Richard Saint-Gelais}

Pas de commencement, pas de milieu, pas de fin, mais des bords...

Thierry Maré

\section{Parmi les blancs}

Entre les cases, il n'y a rien, sinon d'étroits interstices, à gauche et à droite, en haut et en bas, auxquels l'œil du lecteur, d'ordinaire, ne s'arrête pas. Qu'y trouverait-il, d'ailleurs? Quelle action, quel rebondissement, quelle représentation tout court? Aussi les blancs semblent-ils tout naturellement destinés à être sautés: d'où ce mouvement par saccades qui caractérise la lecture de bandes dessinées, faite de contemplations plus ou moins longues de ce qui se trouve dans les cases, séparées par un déplacement infinitésimal et fulgurant vers la prochaine illustration.

«Blancs inter-iconiques": cette appellation proposée par Pierre Fresnault-Deruelle ${ }^{1}$ décrit bien le statut particulier des interstices: les blancs séparent des plages iconiques - les cases - mais ne seraient pas, eux, iconiques. Idée reprise et développée par Michel Rio ${ }^{2}$ : du fait qu'ils sont statutairement hors-fiction, les blancs, écrit-il, constituent chaque fois l'occasion d'une entrée du lecteur dans le domaine de la fiction. Chaque passage d'une case à l'autre apparaît alors comme le passage d'un espace de représentation à un autre, à travers un espace qui lui ne représente rien, mais dont dépend la représentation: l'espace de la page. Celui-ci, ajoute Rio, est nié à chaque nouvelle case, au profit de l'espace imaginaire qui y est figuré; mais cette négation doit constamment être

1 Pierre Fresnault-Deruelle, "Du linéaire au tabulaire ", dans Communications, 24, 1976.

2 Michel Rio, * Cadre, plan, lecture ", ibid. 
recommencée: d'une représentation à l'autre, la lecture doit repasser par un interstice ${ }^{3}$.

Hors-fiction, les blancs seraient aussi, du même coup, métafictionnels: chacun fonctionnerait comme un (discret) index soulignant le caractère fictionnel de ce que la case renferme. La représentation laisse forcément un reste de page (du fait que la $\mathrm{BD}$, strip ou planche, est basée sur le principe de la juxtaposition de plusieurs vignettes), et ce reste se constitue en signes de clôture (blancs, contours des cases) qui désignent, en la délimitant, l'aire que le lecteur est invité à considérer comme un autre espace que celui de la page, un espace régi par d'autres règles, et dont la lecture repose sur des postulats différents - à commencer par la postulation d'une troisième dimension. Lire une planche de $\mathrm{BD}$, c'est passer par intermittence d'un aplat, celui des interstices et des contours, à une profondeur fictive, celle dans laquelle se meuvent les personnages. L'aplat n'est cependant pas une concession à l'espace de la page, une reconnaissance de sa matérialité, mais plutôt un tremplin permettant, paradoxalement, à une partie de cet espace de s'en excepter - on serait tenté de dire: de faire bande à part... - pour se constituer sous le regard du lecteur en un espace fictif qui ne devrait plus rien à celui de la page, qui se soustrairait à ses règles en s'en donnant d'autres. Plus souvent qu'autrement, d'ailleurs, le mouvement de va-et-vient entre le bi- et le tridimensionnel n'est même pas remarqué par celui qui l'effectue. S'il y a métafictionnalité, ici, elle reste donc foncièrement discrète, entièrement absorbée par le réglage de la lecture qu'elle a pour fonction de déclencher. On ne confondra donc pas cette métafictionnalité avec une métafictionnalité ostensible, où l'opération métafictionnelle elle-même ne saurait manquer d'être aperçue par le lecteur (comme c'est le cas par exemple dans certaines cases de Fred ou de Gotlib où les personnages parlent des cases dans lesquelles il se

3 II est temps de signaler que l'interstice peut dans certains cas être remplacé par une juxtaposition des cases, comme souvent chez Bretecher. II arrive aussi, inversement, que le contour des " cases" disparaisse, les blancs inter-iconiques assurant alors seuls la démarcation des espaces de représentation. Ces différences plastiques n'affectent pas, en soi, le principe d'une distinction entre l'espace de la représentation et celui de la page; il s'agirait plutót de différentes façons d'établir cette distinction. 
trouvent) ${ }^{4}$. Sinon, on sacrifie à un effet de mode en voyant de la métafictionnalité partout où le lecteur est invité d'une façon ou d'une autre à considérer quelque chose comme une fiction - ce qui a pour effet de neutraliser les répercussions que ces différentes façons ont en termes de réglage de la lecture. Plus particulièrement, nous perdrions alors la possibilité de distinguer entre les bandes où les interstices appuient l'illusion représentative (l'illusion d'un espace tridimensionnel, notamment) et celles où les interstices travaillent au contraire à faire vaciller la représentation.

\section{Le fantôme du hors-champ: Vaughn-James}

Le fonctionnement des interstices s'apparente, en partie tout au moins, à celui des cadres en général - si ce n'est que les interstices séparent des images disposées les unes à côté des autres, et non des images isolées. D'où cette fonction supplémentaire des interstices par rapport aux autres cadres: ils servent aussi à éviter toute interférence entre les différents espaces de représentation qui se creusent d'une case à l'autre.

C'est que la proximité des cases pourrait fort bien appuyer une lecture désastreuse pour l'écoulement temporel du récit: une lecture qui mettrait toutes les illustrations sur le même plan, et passerait de l'une à l'autre sur la bases de relations spatiales entre elles. Nous savons tous que ce n'est pas le cas: sauter d'une case à l'autre s'accompagne tacitement du postulat, chez le lecteur, qu'un laps de temps (à déterminer via d'autres indices) s'est écoulé entre les deux scènes qui y sont respectivement représentées. Bref: lire une $\mathrm{BD}$, c'est constamment transformer la contiguitté des cases en une consécution des scènes qui y sont représentées. Les interstices ne servent donc pas seulement à établir des restrictions de champ; ils servent aussi à éviter que chaque case ne soit lue comme le hors-champ de quelque autre. Chaque frange, chaque bord, chaque interstice sert donc en principe à éviter toute contamination réciproque des cases,

4 On pourrait dire la même chose, dans un autre domaine, d'une part du mot " roman " sur une page couverture, dispositif métafictionnel mais qui reste éminemment discret, et, d'autre part, d'un énoncé métafictionnel ostensible comme "J'écris Paludes". 
constituant autour de chacune ce qu'on pourrait appeler un fantôme de hors-champ.

En principe, mais en principe seulement, les choses ne sont pas toujours aussi simples. Ce qu'il advient des interstices sous le régime de la représentation ne doit pas être confondu avec ce que les interstices peuvent, dans les faits, déclencher comme processus de lecture. J'examinerai plus loin quelques dispositifs impliquant, soit une bande, soit une planche; j'examinerai d'abord, toutefois, un dispositif reposant sur une image unique.

Soit la page couverture de La cage de Martin VaughnJames (figure 1): une seule image, entourée des mentions habituelles: nom de l'auteur, titre, maison d'édition ${ }^{5}$. Mais ces mentions écrites, justement, sont disposées à l'extérieur de l'image, plutôt que de s'y surimprimer comme il est de coutume dans les couvertures de bandes dessinées. Lillustration, par conséquent, est restreinte à une case, plutôt que de s'étaler en grand comme c'est ordinairement le cas ${ }^{6}$. Mais que montre-t-elle, cette image prise dans un cadrage restreint? Une portion de grillage, devant un paysage d'allure plutôt désertique: de la pierraille qui s'étend jusqu'à l'horizon.

Jouons à affecter à cette une image une fonction illustrative: doit-on y voir la représentation de la cage que le titre annonce? Le cadrage, alors, a ceci d'embarrassant qu'il ne nous laisse qu'un fragment de cage - bref, une cage qui n'emprisonne rien du tout, puisqu'au-delà du grillage l'œil ne rencontre rien jusqu'à l'horizon. Faut-il aussitôt se dire que cette image ne représente pas vraiment une cage? Ne concluons pas si vite: l'autre côté du grillage est libre, soit, mais qu'en est-il de ce côté-ci? Qu'est-ce qui prouve que le terrain désertique ne se trouve pas à l'extérieur de la cage, et que celle-ci ne se referme pas de ce côté-ci du grillage? Le blanc qui entoure l'image prend alors une valeur proprement

5 Martin Vaughn-James, La cage, traduction française de Marc Avelot, Paris, Les impressions nouvelles, 1986.

6 Le lecteur qui ouvrira La cage s'apercevra que ce sont toutes les proportions qui sont inversées: si la couverture se distingue par le petit format de son illustration, les pages intérieures du livre, elles, ne contiennent chacune qu'une seule illustration, beaucoup plus vaste par conséquent que les habituelles vignettes de $B D$. 

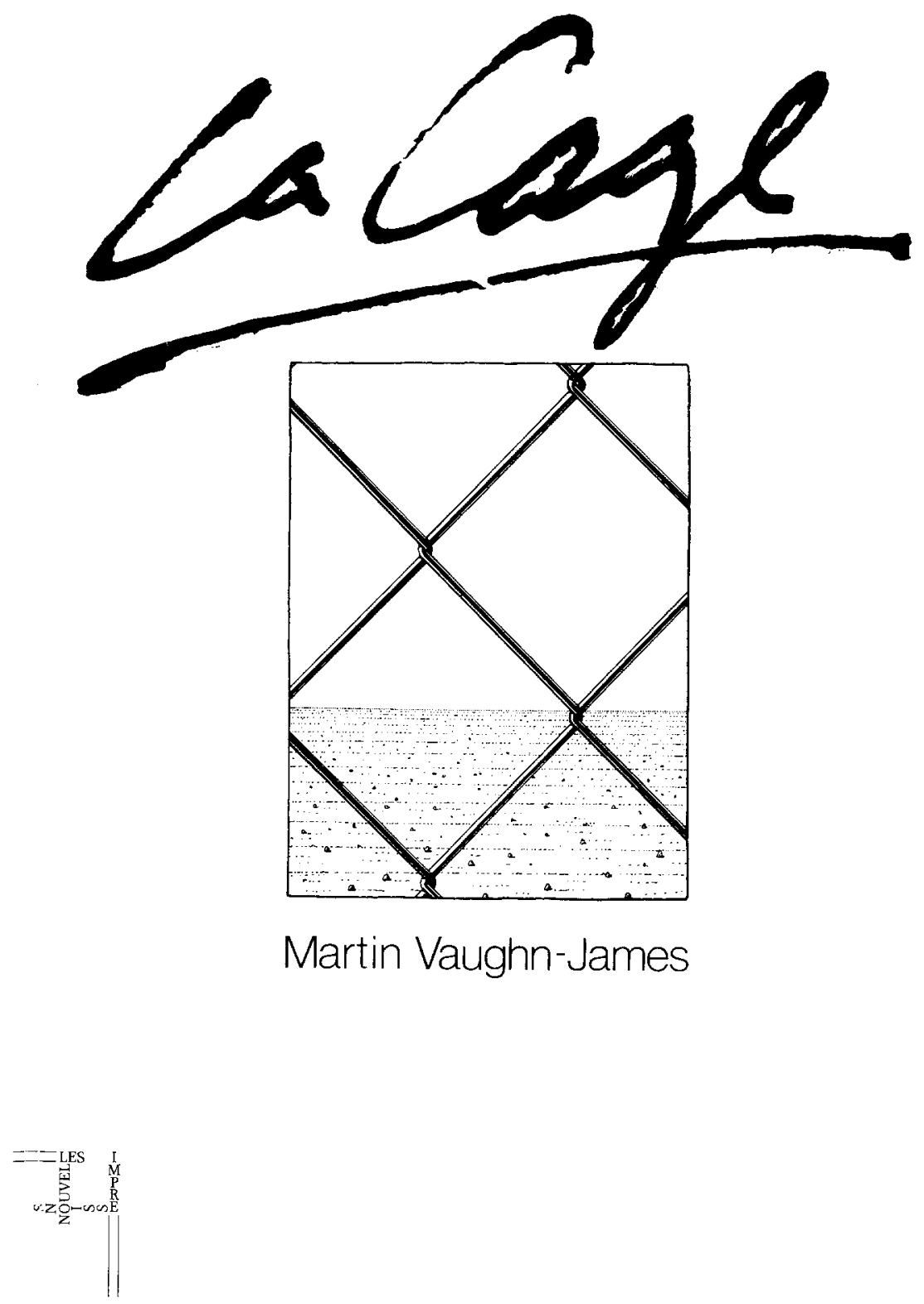

Figure 1 
fictionnelle: il peut alors se lire comme la figuration d'un pâle béton où se découperait une fenêtre grillagée. L'image l'espace de la représentation - ne se limite alors plus à ce que contient le rectangle central: elle absorbe tendanciellement tout l'alentour ${ }^{7}$. Il suffit donc, on le voit, d'un nouveau réglage de la lecture pour que la valeur des signes bascule du non-iconique à l'iconique, et pour que l'image capture ce qui pouvait sembler son cadre. Tout comme elle capture le lecteur lui-même: après tout, qui se trouve placé devant ces barreaux? Dans cette perspective, il n'est pas trop étonnant que le lecteur finisse par s'imaginer comme le prisonnier de cette cage, en train de regarder vers l'extérieur: c'est lui l'acteur de ce retournement des signes, c'est lui qui se "coletaille" avec eux, qui en est soudain proche au point où ceux-ci l'enserrent dans leur réseau. L'« entrée du lecteur dans la fiction", ici, ne se réduit plus à un regard ou à une identification: c'est la personne même du lecteur qui, à la faveur d'une métalepse imprévue, se trouve littéralement prise dans la fiction ${ }^{8}$.

\section{Le retour du hors-champ: Schulz}

En bande dessinée, la case unique n'est évidemment pas le cas le plus typique, de telle sorte que les interstices servent non seulement à indiquer le passage à un espace de représentation, mais aussi, comme on l'a dit, à marquer les frontières entre les différents espaces de représentation, donc à rompre toute continuité spatiale entre les cases, et ce en instaurant une relation autre que spatiale entre elles: une

7 Bien entendu, les signes écrits de la couverture compromettent cette dernière lecture: les mots "La cage" peuvent encore, parce que manuscrits, être lus comme un graffiti, mais le nom d'auteur en caractères d'imprimerie beaucoup moins. En définitive, le dispositif propose, non pas une lecture définitive qui viendrait se substituer à toutes les autres (en l'occurrence, une lecture qui considérerait toute la surface de la couverture comme une illustration), mais plutôt une indétermination qui fait que l'entour de l'image oscille entre l'iconique et le non-iconique. Ou, pour reprendre les termes de Michel Rio, entre l'espace de représentation et l'espace de la page - ce qui montre éloquermment que le premier n'est pas autre chose qu'une lecture particulière du second.

8 Sur la notion de métalepse, voir Gérard Genette, Figures III, Paris, Seuil, 1972 , page 243 et suivantes. 
relation temporelle. Or celle-ci paraît tellement évidente au lecteur, que le passage fictif du temps lui semble la conséquence toute naturelle du passage (lectural) d'une case à l'autre. En fait, cet écoulement n'est pas autre chose que la résultante d'un réglage temporel de la lecture - réglage qui s'accompagne d'une neutralisation de toute relation spatiale entre les cases.

Bien entendu, nombre de bandes dessinées appuient fortement, aussi bien ce réglage temporel que cette neutralisation de toute relation spatiale. Mais ce n'est pas toujours le cas: d'autres effets sont possibles, d'autres dispositifs.
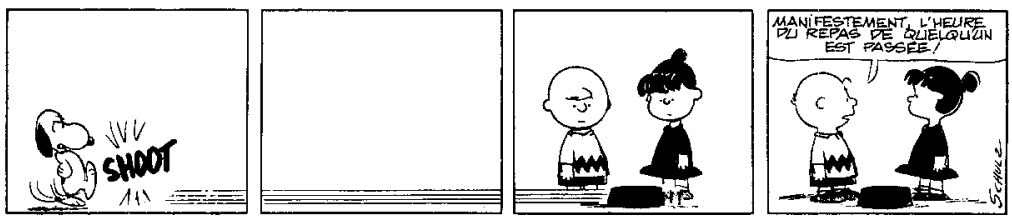

Figure 2

Soit par exemple celui-ci, tiré de la série Peanuts ${ }^{9}$ (figure 2): de la première à la quatrième case, le lecteur peut postuler un écoulement temporel qui fait de chaque case la représentation d'une scène succédant à celle représentée dans la case précédente. Mais ce n'est pas tout: de la case 1 à la case 3 , c'est aussi une relation spatiale qui s'offre à lire: chacune de ces cases présente une scène contiguë à celle des autres cases de la série. On serait donc tenté de dire que cette séquence effectue une sorte de panoramique, allant du coup de patte de Snoopy jusqu'à l'arrivée de la gamelle aux pieds des enfants - mais un panoramique bien différent de ceux qu'on peut voir au cinéma puisqu'il ne s'agit pas d'un écran unique occupé par une image qui bouge, mais bien de trois images segmentées; segmentées, précisément, par des interstices. Ceci a d'ailleurs pour effet de souligner la matérialité de ces dernières, puisque la visible continuité des lignes qui représentent la gamelle en mouvement est par

9 Charles M. Schulz, Incroyable Snoopy, traduction française de J. Dannay, Paris, Dargaud, 1982, p. 17. 
deux fois interrompue. Tout se passe donc comme si la gamelle, pour se rendre du chien aux enfants, devait en quelque sorte traverser l'espace de la page - un espace évidemment hétérogène à celui, tridimensionnel, auquel elle est censée appartenir. Si cette façon de décrire les choses paraît légèrement aberrante (un peu semblable, peut-être, aux voyages de science-fiction à travers l'hyper-espace!), cela tient précisément au dispositif qui est tout près de télescoper deux espaces qui sont censés n'avoir aucun rapport l'un avec l'autre. Censés seulement: l'espace de la représentation ne peut s'édifier que sur (l'oubli de) celui de la page. Le dispositif mis en place par Schulz, certes, n'administre que fort discrètement cette leçon; mais il n'en va pas de même dans certaines planches, beaucoup plus radicales à cet égard, de Fred.

\section{L'espace-temps (paradoxal) de la planche: Fred}

Aux relations temporelles entre cases auxquelles les lecteurs s'en tiennent habituellement, la bande de Schulz parvient à faire superposer (toujours par les lecteurs) des relations de type spatial: les scènes représentées dans les trois premières cases sont à la fois successives et contiguës. Ceci dit, on notera que tout cela se fait sans pour autant mettre en conflit ces deux types de relations qui, dans ce dispositif, s'accommodent l'une de l'autre. Mais il n'en va pas toujours ainsi: il arrive que la lecture se voit amenée à établir et des relations spatiales et des relations temporelles entre cases, mais de telle manière que la coexistence de ces deux types de relations produise toute une série de paradoxes. Fred, l'auteur de la série des Philemon, s'ingénie à élaborer de tels dispositifs où la lecture se prend dans d'inextricables réseaux spatio-temporels.

Je pourrais mentionner sans la montrer une planche, désormais célèbre, de l'album Sinbabbad de Batbad ${ }^{10}$, tant elle a été commentée ${ }^{11}$. L'une des premières choses que le lecteur constate en apercevant cette planche est que les huit cases qui la composent doivent être lues comme composant,

10 Fred, Sinbabbad de Batbad, Paris, Dargaud, 1974, p. 25.

11 Au moins Michel Rio, art. cit., et Benoit Peeters, "Comment lire une BD? ", dans Actes du premier Colloque de BD de Montréal, Montréal, Analogon, 1986. 
simultanément, l'immense corps du chien Sinbabbad: lecture spatialisante, donc ${ }^{12}$. Mais le parcours de case à case montre vite qu'il faut conduire aussi une lecture temporalisante, puisque Philémon, qui apparaît sept fois sur huit cases, se déplace progressivement d'une partie à l'autre du corps du chien ${ }^{13}$. Ces deux lectures entrent en conflit l'une avec l'autre: comme Philémon est figuré à sept reprises, dans autant de cases distinctes, une lecture simultanée de la planche est, logiquement parlant, intenable. Mais l'évidente continuité spatiale des images composant le corps de Sinbabbad fait que cette lecture spatialisante est en même temps inéluctable de telle sorte que le lecteur est amené à accepter une lecture qu'il sait pourtant être une source de contradictions ${ }^{14}$.

Fred pousse cependant la contradiction bien plus loin dans d'autres planches, et notamment dans celle-ci (figure 3) tirée de La mémémoire ${ }^{15}$. Alors que l'espace, dans la planche de Sinbabbad de Batbad, se reconstituait selon une rassurante totalité (seul le parcours est problématique), nous avons ici un dispositif où ni l'espace ni le temps de la fiction ne se laissent penser en termes représentatifs, c'est-à-dire abstraction faite de l'agencement des cases. On s'aperçoit en effet qu'il est possible d'opérer un mouvement de retour entre

12 On dira toutefois du corps de Sinbabbad ce qu'on a dit plus tôt du mouvement de la gamelle dans la bande de Schulz: il est fractionné par les interstices dont la matérialité devient ainsi insistante. Ce qui ne l'empêche toutefois pas d'être recomposable de façon parfaitement cohérente.

13 On notera à la suite de Benoît Peeters (art. cit.) que ce parcours est un parcours impossible, en ce sens qu'on ne peut pas justifier en termes référentiels les positions successivement $\propto$ ccupées par Philémon d'une case à l'autre - séquence pourtant fortement appuyée par la continuité dialoguée. Mais il ne s'agit pas seulement d'un parcours référentiellement invraisemblable: s'il est problématique, c'est que la fiction, pour une fois, subit les répercussions du parcours de la lecture d'une case à l'autre - avec tous les paradoxes que cela implique: d'une case à l'autre. Philémon se retrouve ainsi, dans l'espace de la représentation, là où le mènera le parcours du lecteur à travers l'espace de la page. Fred accentuera ce type de problèmes de façon encore plus spectaculaire dans "Interférence", dans $L$ e fond de l'air est frais..., Paris, Dargaud, 1973. Là, ce ne sont pas seulement les déplacements des personnages dans l'espace, mais aussi la * continuité " dialoguée elle-même qui finit par prendre la configuration d'un labyrinthe inextricable.

14 En ce sens, on a ici affaire à un cas de ce que j'appelle ailleurs une lecture illicite; voir " Les dispositifs illicites (L'Illustre Inconnu et les réglages subversifs de la lecture)", dans Voix et images, $n^{\circ} 44$, hiver 1990.

15 Fred, La mémémoire, Paris, Dargaud, 1977, page 39. 

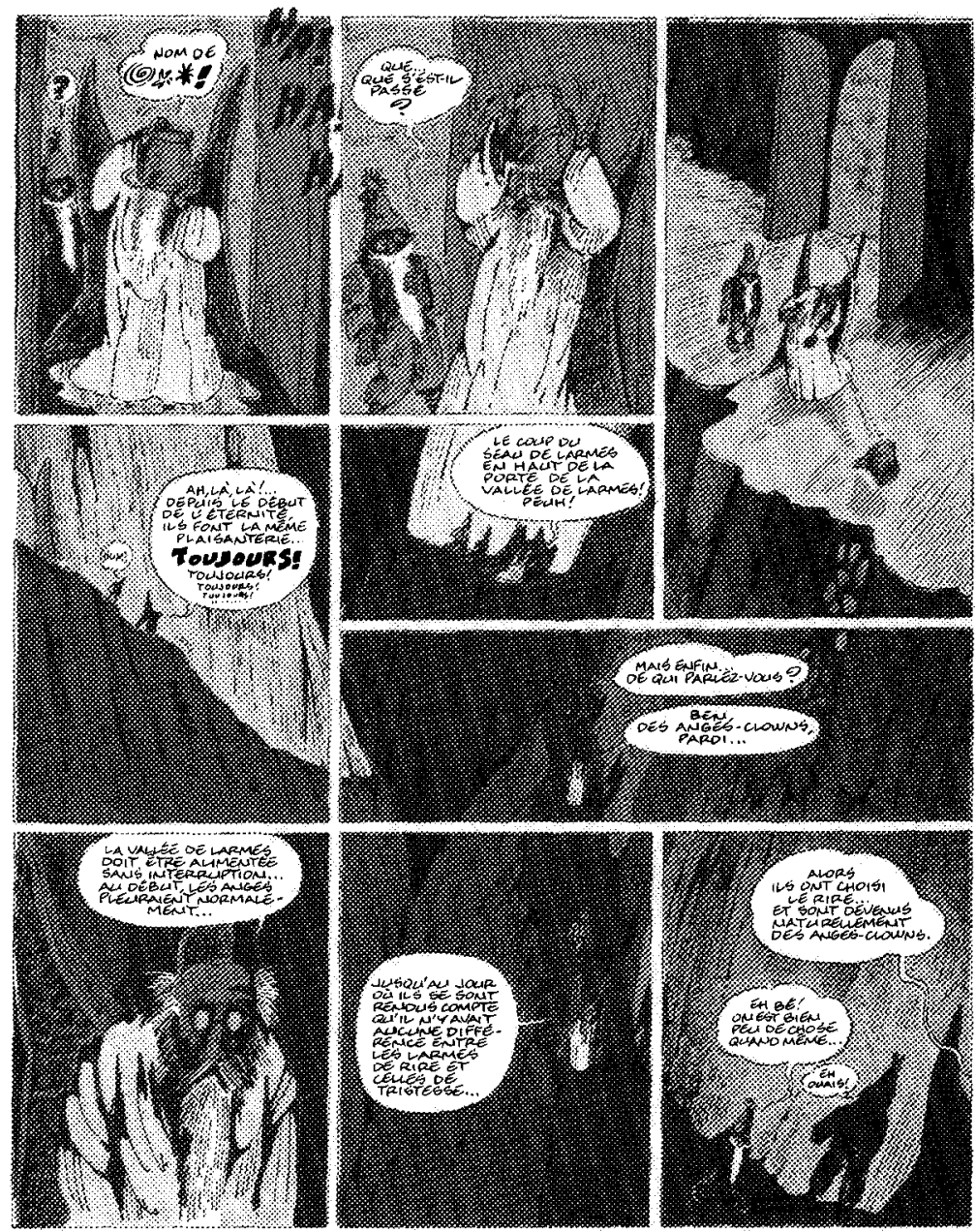

Figure 3 
la case où l'ange dit «Le coup du seau de larmes... » et celle où Barthélémy disait "Que... que s'est-il passé? ?. Or ce mouvement ne se justifie pas par une continuation diégétique de l'action, mais bien par une continuité des tracés et de la couleur (identique dans les deux illustrations). À première vue, on pourrait penser que la situation ressemble à celle où le corps de Sinbabbad se trouve morcelé en plusieurs cases. Mais les choses sont beaucoup moins simples car la continuité plastique (tracés, couleur) s'accompagne en fait d'une incompatibilité de ce qui est représenté: le lecteur passe insensiblement du vêtement de l'ange qui accompagne Barthélémy, à une falaise près de laquelle cet ange et Barthélémy, un peu plus tard, cheminent. Admettre un prolongement entre ces deux cases revient donc à accepter un paradoxe aggravé: non seulement la lecture établit-elle une relation de contiguité entre les cases (ce qui en soi, nous l'avons vu, est plutôt inhabituel en BD), mais de plus l'espace ainsi constitué, vêtement ici, paroi rocheuse là, est foncièrement aberrant. Tout se passe alors comme si le vêtement, passé l'interstice, se muait en falaise - non pas par une transformation fictive (une sorte de pétrification qui ferait partie de l'histoire), mais à partir de la matérialité picturale qui impose ainsi ses bouleversements à l'espace représenté. Espace représenté que, dès lors, on ne peut plus concevoir indépendamment du dispositif constitué par les signes et par leur lecture ${ }^{16}$. Bref: l'espace de la représentation, loin pour une fois de se bâtir sur l'oubli de celui de la page, subit tous les contrecoups d'une lecture pour laquelle la page, ses cases, ses interstices, sont enfin un espace à découvrir et à explorer.

16 Aussi peut-on qualifier de tels dispositifs de a transreprésentatifs " dans le sens que nous donnons ailleurs à ce terme: celui d'un régime de lecture où les éléments représentés ne peuvent pas être pensés abstraction faite des conditions dans lesquels une lecture peut les élaborer. Voir* De la représentation à la transreprésentation ", dans Théories de la lecture/ théories du récit. Actes du colloque intenuniversitaire UQAM-University of Toronto, dans Interprétances, prépublication $n^{\circ} 1,1990$. 